\title{
A PINCHING PROBLEM ON SUBMANIFOLDS WITH PARALLEL MEAN CURVATURE VECTOR FIELD IN A SPHERE
}

\author{
ZHONG HuA Hou
}

\begin{abstract}
Let $M^{n}$ be a closed oriented submanifold with nonzero parallel mean curvature vector field immersed into a unit sphere $S^{n+p}$. Denote by $S$ the square of the length of the second fundamental form. We consider a pinching problem on $S$. We give a pinching constant $C$ on $S$ which depends only on $n$ and $p$. It is better than one given by $\mathrm{Xu}$ [12]. When $p=1,2$ or $n \geq 8$, we show that it is the best possible among this kind of pinching constants. We also characterize those $M^{n}$ with $S=C$.
\end{abstract}

\section{Introduction}

Let $M^{n}$ be a closed oriented submanifold of dimension $n$ with parallel mean curvature vector field immersed into an $(n+p)$-dimensional unit sphere $S^{n+p}$. Denote by $H$ the mean curvature and by $S$ the square of the length of the second fundamental form. We propose to consider the pinching problem on $S$, that is, finding a constant $C$ such that, if $S<C$ on $M^{n}$, then $M^{n}$ is totally umbilical. The constant $C$ so obtained is called the pinching constant of $S$. Moreover, for any $\varepsilon>0$, if there exists an $M^{n}$ in $S^{n+p}$ such that $M^{n}$ is not totally umbilical and $C \leq S<C+\varepsilon$, we say that $C$ is the best possible pinching constant. It is known that, for a closed oriented submanifold of dimension $n$ with parallel mean curvature vector field immersed into an $(n+p)$-dimensional unit sphere $S^{n+p}$, it is totally umbilical if and only if it is an $n$-sphere in $S^{n+p}$.

When $M^{n}$ is minimal, Simons [11] obtained a pinching constant $n /(2-1 / p)$ of $S$ and showed that it can be attained. Chern-do Carmo-Kobayashi [3] and Lawson [6] classified those minimal submanifolds with $S=n /(2-1 / p)$ in $S^{n+p}$. When $p \geq 2$, Li's [7] improved Simons' pinching constant to $2 n / 3$, and showed that it can be attained only by Veronese surface in a totally geodesic $S^{4}$ of $S^{n+p}$.

The pinching problem on $S$ for submanifolds with parallel mean curvature vector field immersed into a sphere was firstly studied by Okumura $[8,9]$. Up to now, there are many remarkable results obtained. The pinching constant depending on $H$ was firstly obtained by Okumura and improved by Alencar-do Carmo [1] (for $p=1$ ) and by $\mathrm{Xu}$ [13] (for $p \geq 1$ ). Since $H$ is a geometric

1991 Mathematics Subject Classification. 53C42, 53A10.

Received July 25, 1997; revised October 21, 1997 
invariant depending on a specific immersion, it is meaningful to give a pinching constant independent of any specific immersions.

The pinching constant depending only on $n$ and $p$ was firstly obtained by Yau [14]. He proved that, for a closed submanifold $M^{n}$ with parallel mean curvature vector field immersed into $S^{n+p}, p>1$, if $S \leq n /\{3+\sqrt{n}-1 /(p-1)\}$, then $M^{n}$ lies in a totally geodesic $S^{n+1}$ of $S^{n+p}$. This result was improved by $\mathrm{Xu}$ [12], who showed that, under the same assumptions as above, if $S \leq$ $\min \{2 n /(1+\sqrt{n}), n /\{2-1 /(p-1)\}\}$, then $M^{n}$ lies in a totally geodesic $S^{n+1}$ of $S^{n+p}$. Furthermore, under additional assumptions, $\mathrm{Xu}$ [12] proved that $M^{n}$ is totally umbilical.

Among all the possible pinching constants depending only on $n$ and $p$, it is significant to find the best possible one. The author [5] showed that $2 \sqrt{n-1}$ is the best possible pinching constant depending on $n$ for $p=1$. In this paper, we will give a pinching constant $C$ of $S$ depending on $n$ and $p$. It is better than the one given by $\mathrm{Xu}$ [12]. When $p=1,2$ or $n \geq 8$, we assert that it is the best possible one among this kind of pinching constants. We also characterize those $M^{n}$ with $S=C$.

Precisely, we propose to prove the following theorems. Denote by $S^{n}(r)$ the standard $n$-dimensional sphere of radius $r$ and define $C$ by

$$
C=\min \left\{2 \sqrt{n-1}, \frac{n}{1+(1 / 2) \operatorname{sgn}(p-2)}\right\}
$$

where $\operatorname{sgn}(\cdot)$ is the standard sign function. Then we have:

THEOREM 1. Let $M^{n}$ be an oriented closed submanifold immersed into the unit sphere $S^{n+p}$, with nonzero parallel mean curvature vector field. If $S<C$, then $S$ is constant and $M^{n}$ is a small sphere $S^{n}(1 / \sqrt{1+S / n})$ in $S^{n+p}$.

THEOREM 2. Let $M^{n}$ be an oriented closed submanifold immersed into the unit sphere $S^{n+p}$, with nonzero parallel mean curvature vector field. Suppose $n>2$ and $S=C$. Then:

(i) If $p=1,2$ or $n \geq 8$, then $C=2 \sqrt{n-1}$ and $M^{n}$ is either a small sphere $S^{n}\left(r_{0}\right)$ in $S^{n+p}$ or a torus $S^{1}(r) \times S^{n-1}(s)$ in a totally geodesic sphere $S^{n+1}$ of $S^{n+p}$, where $r_{0}^{2}=n /(n+2 \sqrt{n-1}), r^{2}=1 /(1+\sqrt{n-1})$ and $s^{2}=\sqrt{n-1} /(1+\sqrt{n-1})$; in $S^{n+p}$.

(ii) If $p>2$ and $n \leq 7$, then $C=2 n / 3$ and $M^{n}$ is a small $n$-sphere $S^{n}(\sqrt{3 / 5})$

THEOREM 3. (i) Let $M^{2}$ be an oriented closed surface immersed into the unit sphere $S^{2+p}$, with nonzero parallel mean curvature vector field. If $p>1$ and $S=C$, then $M^{2}$ is a small sphere $S^{2}(1 / \sqrt{2})(p=2)$ or $S^{2}(\sqrt{3 / 5})(p \geq 3)$ in $S^{2+p}$. that:

(ii) For any $\varepsilon>0$, there exists a pseudo-umbilical surface $M_{\varepsilon}^{2}$ in $S^{2+p}$ such

(a) $M_{\varepsilon}^{2}$ is not totally umbilical;

(b) $M_{\varepsilon}^{2}$ is one with nonzero parallel mean curvature vector field; 
(c) $C<S_{\varepsilon}<C+\varepsilon$, where $S_{\varepsilon}$ is the square of the length of the second fundamental form of $M_{\varepsilon}^{2}$.

Corollary 2. Above theorems show that, when $p=1,2, n=2$ or $n \geq 8, C$ is the best possible pinching constant of $S$ depending only on $n$ and $p$.

We also get a result concerning Erbacher's problem discussed in [3].

Acknowledgments. The author would like to express his hearfelt gratitude to Professor Shūkichi Tanno for continuous encouragement and patient advice during study this subject. He wishes to thank the Okazaki Kaheita International Scholarship Foundation which awarded scholarship to him and standed by him throughout his stay in Japan. Last, the author would be very grateful to referee for their valuable suggestions and constructive criticism.

\section{Formulas of Simons' type}

Let $M^{n}$ be a closed oriented submanifold with nonzero parallel mean curvature vector field immersed into the unit sphere $S^{n+p}$. From now on, we identify $M^{n}$ with its immersed image and agree on the following index ranges:

$$
1 \leq i, j, k, \ldots \leq n ; \quad 1 \leq \alpha, \beta, \gamma, \ldots \leq p ; \quad q \leq A, B, C, \ldots \leq n+p .
$$

Take a local orthonormal frame $\left\{e_{A}\right\}_{A=1}^{n+p}$ in $\mathscr{T}\left(S^{n+p}\right)$ on $M$ such that $\left\{e_{i}\right\}_{l=1}^{n}$ lies in the tangent bundle $\mathscr{T}(M)$ and $\left\{e_{\alpha}\right\}_{\alpha=n+1}^{n+p}$ in the normal bundle $\mathcal{N}(M)$. Let $\left\{\omega_{A}\right\}_{A=1}^{n+p}$ be the dual coframe of $\left\{e_{A}\right\}_{A=1}^{n+p}$. Let $\left(\omega_{A B}\right)_{A, B=1}^{n+p}$ denote the Riemannian connection matrix associated with $\left\{\omega_{A}\right\}_{A=1}^{n+p}$. Then $\left(\omega_{i j}\right)_{i, J=1}^{n}$ defines a Riemannian connection in $\mathscr{T}(\boldsymbol{M})$ and $\left(\omega_{\alpha \beta}\right)_{\alpha, \beta=n+1}^{n+p}$ defines a normal connection in $\mathscr{N}(\boldsymbol{M})$.

It follows that the second fundamental form of $M$ can be expressed as

$$
I I=\sum_{(i, \alpha)} \omega_{i} \otimes \omega_{i \alpha} \otimes e_{\alpha}=\sum_{(i, j, \alpha)} h_{i j}^{\alpha} \omega_{i} \otimes \omega_{j} \otimes e_{\alpha}
$$

where $\omega_{i \alpha}=\sum_{(j)} h_{i j}^{\alpha} \omega_{j}$ and $h_{i j}^{\alpha}=h_{j i}^{\alpha}$ for all $\alpha=n+1, \ldots, n+p$ and $i, j=1, \ldots, n$.

Denote $L_{\alpha}=\left(h_{i j}^{\alpha}\right)_{n \times n}$ and $H_{\alpha}=(1 / n) \sum_{(i)} h_{i i}^{\alpha}$ for $\alpha=n+1, \ldots, n+p$. Then the mean curvature vector field $\xi$ is expressed as $\xi=\sum_{(\alpha)} H_{\alpha} e_{\alpha}$. We denote by $H$ the length of $\xi$ and by $S$ the square of the length of the second fundamental form, i.e., $H=\|\xi\|$ and $S=\sum_{(\alpha, l, j)}\left(h_{i j}^{\alpha}\right)^{2}$. The Riemannian curvature tensor $\left\{R_{i j k l}\right\}$ and the normal curvature tensor $\left\{R_{\alpha \beta k l}\right\}$ are expressed as

$$
R_{i j k l}=\left(\delta_{i k} \delta_{j l}-\delta_{i l} \delta_{j k}\right)+h_{i k}^{\alpha} h_{j l}^{\alpha}-h_{i l}^{\alpha} h_{j k}^{\alpha}, \quad R_{\alpha \beta k l}=h_{k m}^{\alpha} h_{m l}^{\beta}-h_{l m}^{\alpha} h_{m k}^{\beta} .
$$

Define the first and the second covariant derivatives of $\left\{h_{i j}^{\alpha}\right\}$, say $\left\{h_{i j k}^{\alpha}\right\}$ and $\left\{h_{i j k l}^{\alpha}\right\}$ by 


$$
\begin{gathered}
\nabla h_{i j}^{\alpha}=h_{i j k}^{\alpha} \omega_{k} \equiv d h_{i j}^{\alpha}+h_{m j}^{\alpha} \omega_{m i}+h_{i m}^{\alpha} \omega_{m j}+h_{i j}^{\beta} \omega_{\beta \alpha}, \\
\nabla h_{i j k}^{\alpha}=h_{i j k l}^{\alpha} \omega_{l} \equiv d h_{i j k}^{\alpha}+h_{m j k}^{\alpha} \omega_{m i}+h_{i m k}^{\alpha} \omega_{m j}+h_{i j m}^{\alpha} \omega_{m k}+h_{i j k}^{\beta} \omega_{\beta \alpha} .
\end{gathered}
$$

It follows from Ricci's identity that

$$
h_{i j k}^{\alpha}=h_{i k j}^{\alpha}, \quad h_{i j k l}^{\alpha}-h_{i j l k}^{\alpha}=h_{m j}^{\alpha} R_{m i k l}+h_{i m}^{\alpha} R_{m j k l}+h_{i j}^{\beta} R_{\beta \alpha k l} .
$$

The Laplacian of $h_{i j}^{\alpha}$ is defined by $\Delta h_{i j}^{\alpha}=\sum_{(k)} h_{i j k k}^{\alpha}$. Using (3), we have

$$
\begin{aligned}
\Delta h_{i j}^{\alpha}= & h_{k m}^{\alpha} R_{m i j k}+h_{i m}^{\alpha} R_{m k j k}+h_{i k}^{\beta} R_{\beta \alpha j k} \\
= & h_{k m}^{\alpha}\left(\delta_{m j} \delta_{i k}-\delta_{m k} \delta_{i j}+h_{m j}^{\beta} h_{i k}^{\beta}-h_{m k}^{\beta} h_{i j}^{\beta}\right) \\
& +h_{i m}^{\alpha}\left(\delta_{m j} \delta_{k k}-\delta_{m k} \delta_{k j}+h_{m j}^{\beta} h_{k k}^{\beta}-h_{m k}^{\beta} h_{k j}^{\beta}\right) \\
& +h_{i k}^{\beta}\left(h_{j m}^{\beta} h_{m k}^{\alpha}-h_{k m}^{\beta} h_{m j}^{\alpha}\right) \\
= & n h_{i j}^{\alpha}-n H_{\alpha} \delta_{i j}+n H_{\beta} h_{i m}^{\alpha} h_{m j}^{\beta}-S_{\alpha \beta} h_{i j}^{\beta} \\
& +2 h_{i k}^{\beta} h_{k m}^{\alpha} h_{m j}^{\beta}-h_{i m}^{\alpha} h_{m k}^{\beta} h_{k j}^{\beta}-h_{i k}^{\beta} h_{k m}^{\beta} h_{m j}^{\alpha},
\end{aligned}
$$

where we denote $S_{\alpha \beta}=\sum_{(i, j)} h_{i j}^{\alpha} h_{i j}^{\beta}$ for $\alpha, \beta=n+1, \ldots, n+p$.

Define $N(A)=\sum_{(i, j)} a_{i j}^{2}$ for a matrix $A=\left(a_{i j}\right)_{i, j=1}^{n}$ and denote $S_{\alpha}=S_{\alpha \alpha}$ for all $\alpha$. Then we have, for every fixed $\alpha$,

$$
\begin{aligned}
\sum_{(i, j)} h_{i j}^{\alpha} \Delta h_{i j}^{\alpha}= & n S_{\alpha}-n^{2} H_{\alpha}^{2}+n \sum_{(\beta)} H_{\beta} \operatorname{Tr}\left(L_{\alpha}^{2} L_{\beta}\right)-S_{n+1 \alpha}^{2}-\sum_{(\beta>n+1)} S_{\alpha \beta}^{2} \\
& -N\left(L_{\alpha} L_{n+1}-L_{n+1} L_{\alpha}\right)-\sum_{(\beta>n+1)} N\left(L_{\alpha} L_{\beta}-L_{\beta} L_{\alpha}\right) .
\end{aligned}
$$
have

Choose $e_{n+1}$ to have the same direction as $\xi$ such that $\xi=H e_{n+1}$. Then we

$$
H_{n+1}=H ; \quad H_{\alpha}=0, \quad \alpha=n+2, \ldots, n+p .
$$

Since $\xi$ is nonzero and parallel, we have that $H \neq 0$ is constant and $e_{n+1}$ is parallel. It follows that $L_{n+1} L_{\alpha}=L_{\alpha} L_{n+1}$. From (4), we obtain

$$
\begin{aligned}
\sum_{(i, j)} h_{i j}^{n+1} \Delta h_{i j}^{n+1}= & n S_{n+1}+n H \operatorname{Tr}\left(L_{n+1}\right)^{3}-n^{2} H^{2}-S_{n+1}^{2}-\sum_{(\beta>n+1)}\left(S_{n+1 \beta}\right)^{2} \\
\sum_{(i, j)} h_{i j}^{\alpha} \Delta h_{i j}^{\alpha}= & n S_{\alpha}+n H \operatorname{Tr} L_{n+1}\left(L_{\alpha}\right)^{2}-\left(S_{n+1 \alpha}\right)^{2}-\sum_{(\beta>n+1)}\left(S_{\alpha \beta}\right)^{2} \\
& -\sum_{(\beta>n+1)} N\left(L_{\beta} L_{\alpha}-L_{\alpha} L_{\beta}\right), \quad \alpha>n+1 .
\end{aligned}
$$

We recall that a submanifold is said to be pseudo-umbilical if the mean curvature vector field is nonzero and lies in an umbilical direction of the 
fundamental form. Define $\tilde{S}_{n+1}$ by

$$
\tilde{S}_{n+1}=\sum_{(i, j)}\left(h_{i j}^{n+1}-H \delta_{i j}\right)^{2} .
$$

It is easy to get the following

Lemma 1. Let $\tilde{S}_{n+1}$ be defined as in (**). Then $\tilde{S}_{n+1}=S_{n+1}-n H^{2} \geq 0$ and the equality holds if and only if $M^{n}$ is pseudo-umbilical.

We denote $f=\operatorname{Tr}\left(L_{n+1}\right)^{3}$ and $S_{I}=\sum_{(\beta>n+1)} S_{\beta}$. It follows from (6) that

$$
\sum_{(i, j)} h_{i j}^{n+1} \Delta h_{i j}^{n+1}=n S_{n+1}+n H f-n^{2} H^{2}-S_{n+1}^{2}-\sum_{(\beta>n+1)}\left(S_{n+1 \beta}\right)^{2} .
$$

Using the same arguments as in [5], we have

$$
\begin{aligned}
\sum_{(i, j)} h_{i j}^{n+1} \Delta h_{i j}^{n+1} \geq & \tilde{S}_{n+1}\left\{n-\left(\tilde{S}_{n+1}-n H^{2}\right)-\frac{n-2}{\sqrt{n-1}} H \sqrt{n \tilde{S}_{n+1}}\right\} \\
& -\sum_{(\beta>n+1)}\left(S_{n+1 \beta}\right)^{2} .
\end{aligned}
$$

It follows from (5) that

$$
\sum_{(\beta>n+1)}\left(S_{n+1 \beta}\right)^{2}=\sum_{(\beta>n+1)}\left\{\sum_{(i, j)}\left(h_{i j}^{n+1}-H \delta_{i j}\right) h_{i j}^{\beta}\right\}^{2} .
$$

By applying Schwarz's inequality to the right hand-side of (10), we have

$$
\sum_{(\beta>n+1)}\left(S_{n+1 \beta}\right)^{2} \leq \tilde{S}_{n+1} S_{I}
$$

Substituting (11) into (9), we have

$$
\begin{aligned}
\sum_{(i, j)} h_{i j}^{n+1} \Delta h_{i j}^{n+1} & \geq \tilde{S}_{n+1}\left\{n-\left(\tilde{S}_{n+1}-n H^{2}\right)-S_{I}-\frac{n-2}{\sqrt{n-1}} H \sqrt{n \tilde{S}_{n+1}}\right\} \\
& =\tilde{S}_{n+1}\left\{n-\tilde{S}+n H^{2}-\frac{n-2}{\sqrt{n-1}} H \sqrt{n \tilde{S}_{n+1}}\right\} \\
& \geq \tilde{S}_{n+1}\left\{n-\tilde{S}+n H^{2}-\frac{n-2}{\sqrt{n-1}} H \sqrt{n \tilde{S}}\right\},
\end{aligned}
$$

where $\tilde{S}=\tilde{S}_{n+1}+S_{I}=S-n H^{2}$.

Using the same arguments as in [5] to the last term of (12), we obtain

$$
\sum_{(i, j)} h_{i j}^{n+1} \Delta h_{i j}^{n+1} \geq \tilde{S}_{n+1}\left(n-\frac{n}{2 \sqrt{n-1}} S\right) .
$$


It follows that

$$
\frac{1}{2} \Delta S_{n+1}=\sum_{(i, j, k)}\left(h_{i j k}^{n+1}\right)^{2}+\sum_{(i, j)} h_{i j}^{n+1} \Delta h_{i j}^{n+1} \geq \tilde{S}_{n+1}\left(n-\frac{n}{2 \sqrt{n-1}} S\right) .
$$

Taking integrations on both sides of (13) on $M^{n}$, we obtain

$$
0 \geq \int_{M^{n}} \tilde{S}_{n+1}\left(n-\frac{n}{2 \sqrt{n-1}} S\right) .
$$

If $S \leq 2 \sqrt{n-1}$, we have from (13), (14) and Hopf's Lemma that $S_{n+1}$ is constant and

$$
\tilde{S}_{n+1}\left(n-\frac{n}{2 \sqrt{n-1}} S\right)=0 \text {. }
$$

It follows from Lemma that $\tilde{S}_{n+1}$ is also a constant. Therefore we obtain the following

PROPOSITION 1. Let $M^{n}$ be a closed submanifold immersed into a unit sphere $S^{n+p}$, with nonzero parallel mean curvature vector field. If $S \leq 2 \sqrt{n-1}$, then we have:

(i) $S=2 \sqrt{n-1}$; or

(ii) $\tilde{S}_{n+1}=0$ and $M^{n}$ is pseudo-umbilical.

If $M^{n}$ is not pseudo-umbilical, we have from (12) that $S_{I} \equiv 0$. It follows that $M^{n}$ lies in a totally geodesic sphere $S^{n+1}$ of $S^{n+p}$. From a result in [5], we get the following

COROllary 1. Under the same assumptions as in Proposition 1, we have:

(i) Suppose $n>2$. If $M^{n}$ is not pseudo-umbilical and $S \leq 2 \sqrt{n-1}$, then $S=2 \sqrt{n-1}$ and $M^{n}$ is a torus $S^{1}(r) \times S^{n-1}(s)$ in a totally geodesic sphere $S^{n+1}$ of $S^{n+p}$, where $r^{2}=1 /(1+\sqrt{n-1})$ and $s^{2}=\sqrt{n-1} /(1+\sqrt{n-1})$;

(ii) Suppose $n=2$. If $S \leq 2$, then $M^{2}$ is pseudo-umbilical.

Sketch of the proof of Corollary 1. (i) is obvious from [5]. To prove (ii), we need only to consider the case $S=2$. Supposing that $M^{2}$ is not umbilical, we have that $M^{2}$ can be immersed as a flat torus $S^{1}(r) \times S^{1}(s)$ into a totally geodesic sphere $S^{3}$ in $S^{p+2}$. But the only flat torus with $S=2$ in $S^{3}$ is the Clifford torus, which is minimal. This contradicts the assumption $H \neq 0$. We complete the proof.

Q.E.D.

From now on, we suppose that $M^{n}$ is pseudo-umbilical and $p \geq 2$. In this case, we know that $M^{n}$ can be minimally immersed into a hypersphere $S^{n+p-1}\left(1 / \sqrt{1+H^{2}}\right)$ of $S^{n+p}$. 
Chen [2] proved the following classification result (see also Santos [10, pp. 411]): Let $M^{n}$ be a compact pseudo-umbilical submanifold of $S^{n+p}, p \geq 2$, with parallel mean curvature vector field. If $S \leq n\left(1+H^{2}\right) /\{2-1 /(p-1)\}$, then either (i) $S=0$ and $M^{n}$ is totally umbilical; or (ii) $S=n\left(1+H^{2}\right) /\{2-1 /(p-1)\}$ and $M^{n}$ is a minimal Clifford hypersurface in $S^{n+1}\left(1 / \sqrt{1+H^{2}}\right) \hookrightarrow S^{n+2}$ or $M^{2}$ is a Veronese surface in $S^{4}\left(1 / \sqrt{1+H^{2}}\right) \hookrightarrow S^{5}$.

We propose to give an improvement to this result.

Since $L_{n+1}=H I_{n}$ in this case, we have, from (10),

$$
\sum_{(\beta>n+1)}\left(S_{n+1 \beta}\right)^{2}=0 .
$$

It follows from (7) and (15) that

$$
\sum_{(i, j ; \alpha>n+1)} h_{i j}^{\alpha} \Delta h_{i j}^{\alpha}=n\left(1+H^{2}\right) S_{I}-\sum_{(\alpha, \beta>n+1)}\left(S_{\alpha \beta}\right)^{2}-\sum_{(\alpha, \beta>n+1)} N\left(L_{\beta} L_{\alpha}-L_{\alpha} L_{\beta}\right) .
$$

We have to estimate the sum of the last two terms in the right-hand side of (16).

Li's [7] proved the following

LeMma 2. Let $A_{1}, A_{2}, \ldots, A_{q}$ be symmetric $(n \times n)$-matrices, where $q \geq 2$. We denote $S_{\alpha \beta}=\operatorname{Tr} A_{\alpha}^{T} A_{\beta}, S_{\alpha}=S_{\alpha \alpha}=N\left(A_{\alpha}\right)$ and $S=S_{1}+\cdots+S_{q}$. Then

$$
\sum_{(\alpha, \beta)} S_{\alpha \beta}^{2}+\sum_{(\alpha, \beta)} N\left(A_{\beta} A_{\alpha}-A_{\alpha} A_{\beta}\right) \leq \frac{3}{2} S^{2},
$$

and the equality holds if and only if one of the following conditions holds:

(i) $A_{1}=\cdots=A_{q}=0$;

(ii) only two of the matrices $A_{1}, A_{2}, \ldots, A_{q}$ are different from zero. Moreover, assuming $A_{1} \neq 0, A_{2} \neq 0$ and $A_{3}=\cdots=A_{q}=0$, we have $S_{1}=S_{2}$ and there exists an orthogonal $(n \times n)$-matrix $U$ such that

$$
U A_{1} U^{T}=\sqrt{\frac{S_{1}}{2}}\left(\begin{array}{cc|c}
1 & 0 & 0 \\
0 & -1 & \\
\hline 0 & 0
\end{array}\right), \quad U A_{2} U^{T}=\sqrt{\frac{S_{1}}{2}}\left(\begin{array}{cc|c}
0 & 1 & 0 \\
1 & 0 & \\
\hline 0 & 0
\end{array}\right) .
$$

Together with the case $q=1$, we obtain

$$
\sum_{(\alpha, \beta)} S_{\alpha \beta}^{2}+\sum_{(\alpha, \beta)} N\left(A_{\beta} A_{\alpha}-A_{\alpha} A_{\beta}\right) \leq[1+(1 / 2) \operatorname{sgn}(q-1)] S^{2} .
$$

Replacing $A_{\alpha}$ 's in (18) by $L_{\beta}$ 's with $\beta>n+1$, we have

$$
\sum_{(\alpha, \beta<n+1)} S_{\alpha \beta}^{2}+\sum_{(\alpha, \beta>n+1)} N\left(L_{\beta} L_{\alpha}-L_{\alpha} L_{\beta}\right) \leq[1+(1 / 2) \operatorname{sgn}(p-2)] S_{I}^{2} .
$$


Substituting above inequality into (16), we obtain

$$
\sum_{(i, j ; \alpha>n+1)} h_{i j}^{\alpha} \Delta h_{i j}^{\alpha} \geq S_{I}\left\{n\left(1+H^{2}\right)-[1+(1 / 2) \operatorname{sgn}(p-2)] S_{I}\right\} .
$$

It follows that

$$
\begin{aligned}
\frac{1}{2} \Delta S_{I} & =\sum_{(i, j, k ; \alpha>n+1)}\left(h_{i j k}^{\alpha}\right)^{2}+\sum_{(i, j ; \alpha>n+1)} h_{i j}^{\alpha} \Delta h_{i j}^{\alpha} \\
& \geq S_{I}\left\{n\left(1+H^{2}\right)-[1+(1 / 2) \operatorname{sgn}(p-2)] S_{I}\right\} .
\end{aligned}
$$

Using (19), we can improve Chen's result as follows:

Proposition 2. Let $M^{n}$ be a closed pseudo-umbilical submanifold with nonzero parallel mean curvature vector field immersed into a unit sphere $S^{n+p}$ with $p \geq 2$. Then $M^{n}$ can be minimally immersed into a hypersphere $S^{n+p-1}\left(1 / \sqrt{1+H^{2}}\right)$ in $S^{n+p}$. Furthermore, if $S_{I} \leq n\left(1+H^{2}\right) /[1+(1 / 2) \operatorname{sgn}(p-2)]$, we have:

(i) $S_{I}=0$ and $M^{n}$ is a small sphere $S^{n}\left(1 / \sqrt{1+H^{2}}\right)$ in $S^{n+p}$;

(ii) $S_{I}=n\left(1+H^{2}\right)$ and $M^{n}$ is a Clifford torus $S^{k}(r) \times S^{n-k}(s)$ in a hypersphere $S^{n+1}\left(1 / \sqrt{1+H^{2}}\right)$ in a totally geodesic sphere $S^{n+2}$ of $S^{n+p}$;

(iii) $S_{I}=(4 / 3)\left(1+H^{2}\right)$ and $M^{2}$ is the Veronese surface in a hypersphere $S^{4}\left(1 / \sqrt{1+H^{2}}\right)$ in a totally geodesic sphere $S^{5}$ of $S^{n+p}$.

Proof. It is clear that $M^{n}$ can be minimally immersed into a hypersphere $S^{n+p-1}\left(1 / \sqrt{1+H^{2}}\right)$ in $S^{n+p}$. Let us prove assertions (i)-(iii).

First, we have to show that (19) works on the reduced immersion.

Recall that the normal connection matrix of $M^{n}$ in $S^{n+p}$ is $\left(\omega_{\alpha \beta}\right)_{\alpha, \beta=n+1}^{n+p}$. Hence the normal connection matrix of $M^{n}$ in $S^{n+p-1}\left(1 / \sqrt{1+H^{2}}\right)$ can be expressed as $\left(\omega_{\alpha \beta}\right)_{\alpha, \beta=n+2}^{n+p}$ and the square of the length of the second fundamental form of $M^{n}$ in $S^{n+p-1}\left(1 / \sqrt{1+H^{2}}\right)$ is the same as the $S_{I}$ of $M^{n}$ in $S^{n+p}$. On the other hand, we have $\omega_{n+1 \alpha}=0, \alpha=n+1, \ldots, n+p$, since the mean curvature vector field $\xi=H e_{n+1}$ is parallel in the normal bundle $\mathscr{N}(M)$. Hence the covariant derivatives of $\left\{h_{i j}^{\alpha}\right\}$ in $S^{n+p-1}\left(1 / \sqrt{1+H^{2}}\right)$ are the same as that of $\left\{h_{i j}^{\alpha}\right\}$ in $S^{n+p}$. And so is the Laplacian of $\left\{h_{i j}^{\alpha}\right\}$.

Therefore (19) can also be considered as being computed on the minimal immersion from $M^{n}$ into $S^{n+p-1}\left(1 / \sqrt{1+H^{2}}\right)$ of constant curvature $\left(1+H^{2}\right)$.

Taking integration on both-sides of (19) on $M^{n}$, we have

$$
0 \geq \int_{M^{n}} S_{I}\left\{n\left(1+H^{2}\right)-[1+(1 / 2) \operatorname{sgn}(p-2)] S_{I}\right\}
$$

From $\left(19^{\prime}\right)$ and the assumption, we have $S_{I}=0$ or $S_{I}=n\left(1+H^{2}\right) /$ $[1+(1 / 2) \operatorname{sgn}(p-2)]$, on $M^{n}$. Assertion (i) follows directly from $S_{I}=0$. If 
$p=2$, then $S_{I}=n\left(1+H^{2}\right)$. Assertion (ii) follows from the result of Cherndo Carmo-Kobayashi [3]. If $p \geq 3$, then $S_{I}=(4 / 3)\left(1+H^{2}\right)$. Following the same arguments as in Li's [7], we obtain assertion (iii). This completes the proof.

Q.E.D.

Remark 1. From $\left(19^{\prime}\right)$, we can immediately get

$$
0 \geq \int_{M^{n}} S_{I}\{n-[1+(1 / 2) \operatorname{sgn}(p-2)] S\}
$$

which will be used in next section.

\section{Proof of the theorems}

Let $C$ be defined as in (*). It is easy to see that

$$
4(n-1)-\frac{4}{9} n^{2}=-\frac{4}{9}[(n-2)(n-7)-5] \begin{cases}>0, & 2 \leq n \leq 7 \\ <0, & 8 \leq n\end{cases}
$$

Therefore we have

$$
C=\left\{\begin{array}{lll}
2 \sqrt{n-1}, & p=2 & \text { or } 8 \leq n \\
2 n / 3, & p>2 & \text { and } 2 \leq n \leq 7
\end{array}\right.
$$

If $S<C$, then $S<2 \sqrt{n-1}$. From Proposition 1 , we have that $M^{n}$ is pseudo-umbilical. In this case, it follows from (20) that $S_{I} \equiv 0$. Hence $S=$ $S_{I}+n H^{2}=n H^{2}$. Using Proposition 2, we can see that $M^{n}$ is a small sphere $S^{n}(1 / \sqrt{1+S / n})$ in $S^{n+p}$.

Therefore we obtain the following

THEOREM 1. Let $M^{n}$ be an oriented closed submanifold immersed into the unit sphere $S^{n+p}$, with nonzero parallel mean curvature vector field. If $S<C$, then $S$ is constant and $M^{n}$ is a small sphere $S^{n}(1 / \sqrt{1+S / n})$ in $S^{n+p}$.

Now let us consider the case that $M^{n}$ is one with $S=C$.

Case 1. Suppose $n>2$. If $p=2$ or $n \geq 8$, then $C=2 \sqrt{n-1}<n$ / $[1+1 / 2 \operatorname{sgn}(p-2)]$. From (20) we have $S_{I} \equiv 0$. If $M^{n}$ is not pseudo-umbilical, it follows from Corollary 1 that $M^{n}$ is a torus $S^{1}(r) \times S^{n-1}(s)$ in a totally geodesic sphere $S^{n+1}$ of $S^{n+p}$ where $r^{2}=1 /(1+\sqrt{n-1})$ and $s^{2}=\sqrt{n-1} /(1+\sqrt{n-1})$. If $M^{n}$ is pseudo-umbilical, it follows from Proposition 2 that $M^{n}$ is a small sphere $S^{n}\left(r_{0}\right)$ in $S^{n+p}$ where $r_{0}^{2}=n /(n+2 \sqrt{n-1})$.

If $p>2$ and $n \leq 7$, we have $C=n /[1+1 / 2 \operatorname{sgn}(p-2)]<2 \sqrt{n-1}$. Therefore $M^{n}$ is pseudo-umbilical. In this case $S_{I}<S=2 n / 3<n\left(1+H^{2}\right) /$ $[1+1 / 2 \operatorname{sgn}(p-2)]$. It follows from Proposition 2 that $S_{I}=0$ and $M^{n}$ is a small sphere $S^{n}(\sqrt{3 / 5})$ in $S^{n+p}$. 
From the above discussions plus the case $p=1$, we obtain the following

THEOREM 2. Let $M^{n}$ be an oriented closed submanifold immersed into the unit sphere $S^{n+p}$, with nonzero parallel mean curvature vector field. Suppose $n>2$ and $S=C$. Then:

(i) If $p=1,2$ or $n \geq 8$, then $C=2 \sqrt{n-1}$ and $M^{n}$ is either a small sphere $S^{n}\left(r_{0}\right)$ in $S^{n+p}$ or a torus $S^{1}(r) \times S^{n-1}(s)$ in a totally geodesic sphere $S^{n+1}$ of $S^{n+p}$, where $r_{0}^{2}=n /(n+2 \sqrt{n-1}), r^{2}=1 /(1+\sqrt{n-1})$ and $s^{2}=\sqrt{n-1} /(1+\sqrt{n-1})$; in $S^{n+p}$.

(ii) If $p>2$ and $n \leq 7$, then $C=2 n / 3$ and $M^{n}$ is a small $n$-sphere $S^{n}(\sqrt{3 / 5})$

Case 2. Suppose $n=2$ and $p \geq 2$. If $p=2$, then $C=2<2\left(1+H^{2}\right)$. From (i) of Proposition 2 we have that $M^{2}$ is a sphere $S^{2}(1 / \sqrt{2})$.

If $p>2$, then $C=4 / 3<2$. It follows from Corollary 1 that the $M^{2}$ with $S=C$ is pseudo-umbilical. Thus $M^{2}$ can be minimally immersed into a hypersphere $S^{p+1}\left(1 / \sqrt{1+H^{2}}\right)$ of $S^{p+2}$. Note that $S_{I}<S=4 / 3<4\left(1+H^{2}\right) / 3$. From (i) of Proposition 2 we have that $M^{2}$ is a sphere $S^{2}(\sqrt{3 / 5})$.

For an arbitrary $\varepsilon>0$, we can choose $H_{\varepsilon}$ small enough such that

Then we have

$$
\left(1+1 /\left[1+\frac{1}{2} \operatorname{sgn}(p-2)\right]\right) \cdot 2 H_{\varepsilon}^{2}<\varepsilon
$$

$$
C<S_{\varepsilon}=S_{I}+2 H_{\varepsilon}^{2}=2\left(1+H_{\varepsilon}^{2}\right) /\left[1+\frac{1}{2} \operatorname{sgn}(p-2)\right]+2 H_{\varepsilon}^{2}<C+\varepsilon .
$$

From (ii) of Proposition 2, we can see that the only minimal surface with $S_{I}=2\left(1+H_{\varepsilon}^{2}\right)$, in a hypersphere $S^{3}\left(1 / \sqrt{1+H_{\varepsilon}^{2}}\right)$ of a totally geodesic sphere $S^{4}$ of $S^{2+p}$, is the Clifford torus $S^{1}\left(1 / \sqrt{2\left(1+H_{\varepsilon}^{2}\right)}\right) \times S^{1}\left(1 / \sqrt{2\left(1+H_{\varepsilon}^{2}\right)}\right)$. From (iii) of Proposition 2, we can see that the only minimal surface with $S_{I}=$ $4\left(1+H_{\varepsilon}^{2}\right) / 3$, in a hypersphere $S^{4}\left(1 / \sqrt{1+H_{\varepsilon}^{2}}\right)$ of a totally geodesic sphere $S^{5}$ of $S^{2+p}$, is the Veronese surface.

Therefore we obtain the following.

THEOREM 3. (i) Let $M^{2}$ be an oriented closed surface immersed into the unit sphere $S^{2+p}$, with nonzero parallel mean curvature vector field. If $p>1$ and $S=C$, then $M^{2}$ is a small sphere $S^{2}(1 / \sqrt{2})(p=2)$ or $S^{2}(\sqrt{3 / 5})(p \geq 3)$ in $S^{2+p}$. that:

(ii) For any $\varepsilon>0$, there exists a pseudo-umbilical surface $M_{\varepsilon}^{2}$ in $S^{2+p}$ such

(a) $M_{\varepsilon}^{2}$ is not totally umbilical;

(b) $M_{\varepsilon}^{2}$ is one with nonzero parallel mean curvature vector field;

(c) $C<S_{\varepsilon}<C+\varepsilon$, where $S_{\varepsilon}$ is the square of the length of the second fundamental form of $M_{\varepsilon}^{2}$.

Therefore we can claim our desired conclusion: 
Corollary 2. Above theorems show that, when $p=1,2, n=2$ or $n \geq 8$, $C$ is the best possible pinching constant of $S$ depending only on $n$ and $p$.

Erbacher [4] suggested the following problem:

When can we reduce the codimension of an isometric immersion into a space form of constant curvature?

and got a result under an assumption on the first normal space of the isometric immersion.

From Theorems 1-3, we can get the following

Proposition 3. Let $M^{n}$ be a closed submanifold immersed into the unit sphere $S^{n+p}$ with nonzero parallel mean curvature vector field. If $S \leq C$, then the codimension $p$ of $M^{n}$ can be reduced to one.

\section{REFERENCES}

[1] H. Alencar AND M. P. Do CaRmo, Hypersurfaces with constant mean curvature in spheres, Proc. Amer. Math. Soc., 120 (1994), 1223-1229.

[2] S. P. ChEN, Pseudo-umbilical submanifolds in spaces of constant curvature, J. East. China Norm. Univ. Natur. Sci. Ed., 1 (1989), 59-65 (in Chinese).

[ 3 ] S. S. Chern, M. do Carmo and S. Kobayashn, Minimal submanifolds of a sphere with second fundamental form of constant length, Functional Analysis and Related Fields, Springer, New York, 1970, 59-75.

[4] J. ERBACHER, Reduction of the codimension of an isometric immersion, J. Differential Geom., 5 (1971), 333-340.

[5] Z. H. Hou, Hypersurfaces in sphere with constant mean curvature, Proc. Amer. Math. Soc., 125 (1997), 1193-1196.

[6] H. B. LAwson, JR., Local rigidity theorems for minimal hypersurfaces, Ann. of Math. (2), 89 (1969), 187-197.

[7] A. M. LI AND J. M. LI, An intrinsic rigidity theorem for minimal submanifolds in a sphere, Arch. Math. (Basel), 58 (1992), 582-594.

[8] M. OKumuRA, Submanifolds and a pinching problem on the second fundamental tensor, Trans. Amer. Math. Soc., 178 (1973), 285-291.

[9] M. OKumura, Hypersurfaces and a pinching problem on the second fundamental tensor, Amer. J. Math., 96 (1974), 207-213.

[10] W. SANTos, Submanifolds with parallel mean curvature vector in spheres, Tôhoku Math. J., 46 (1994), 403-415.

[11] J. Simons, Minimal varieties in Riemannian manifolds, Ann. of Math. (2), 88 (1968), 62-105.

[12] H. W. XU, A pinching constant of Simons' type and isometric immersions, Chinese Ann. Math. Ser. A, 12 (1991), 261-269.

[13] H. W. XU, A rigidity theorem for submanifolds with parallel mean curvature in a sphere, Arch. Math. (Basel), 61 (1993), 489-496.

[14] S. T. YAU, Submanifolds with constant mean curvature I, Amer. J. Math., 96 (1975), 346366; II, Amer. J. Math., 97 (1975), 76-100.

DEPARTMENT OF MATHEMATICS

TOKYo INSTITUTE OF TEChNOLOGY

Oh-okayama, Meguro-Ku

ToKYo 152-8551, JAPAN

E-mail: hou@math.titech.ac.jp 